\title{
Speed Up of Shape from Shading Using Graduated Non-convexity
}

\author{
Daniele Gelli and Domenico Vitulano \\ Istituto per le Applicazioni del Calcolo IAC-C.N.R. \\ Viale del Policlinico 137, 00161 Roma, Italy \\ \{Gelli,Vitulano\}@iac.rm.cnr.it
}

\begin{abstract}
This paper will focus on a proposal to speed up Shape From Shading (SFS) approaches based on energy minimization. To this end, Graduated Non Convexity (GNC) algorithm has been adopted to minimize this strongly non convex energy. Achieved results are very promising and involve aspects both theoretical and practical. In fact, both a generalization of the original formulation of GNC and an effective discrete shape recovery characterize our approach. Finally, a drastic reduction of the computational time is reached in comparison with the other currently available approaches.
\end{abstract}

\section{Introduction}

Shape From Shading (SFS) is a classical problem of Computer Vision and consists of recovering 3-D shape of an object starting from its image 112. In the last few years a lot of research has been devoted to it. The approaches in literature can be coarsely classified in four groups: minimization, propagation, local and linear.

Minimization based approaches try to achieve the solution via minimization of an energy composed of some terms: each of them corresponds to a constraint on the solution itself 3.4. In the second class, introduced by Horn [3] and later developed by Bruckstein [5], shape information is propagated along the strips in the direction of intensity gradient. These latter are lines in the image where both surface depth and orientation can be computed whether they are known at the starting point. New approaches are based on Hamilton Jacobi equation and viscosity solution, such as [6] and the one recently proposed in 7], where Eikonal equation is solved using Fast Marching Method. Local approaches exploit some "a priori" assumptions on the shape to be recovered (e.g. locally spherical as in [8]). Finally, approaches belonging to the last class are based on linearization of the SFS scheme to achieve the surface depth. Two examples are in 19].

Minimization approaches seem to achieve better results among the aforementioned ones [2]. Their only drawback consists of a huge computing time in reaching the solution. Then they are not suitable for real time applications. This problem stems from the fact that involved energies are usually not convex functions. Hence, their (global) minimum is hard to be found. 
This paper focuses on a drastic reduction of the SFS computing time using Graduated Non Convexity (GNC) approach [10]. The latter is a clever method for minimizing discrete non convex energies. It consists of producing a sequence of energy functions, by a gradual deconvexification of the original one. This way allows us to reach a minimum close to the absolute one, in a moderate computing time [10. If on one hand $G N C$ is a valid alternative to very expensive algoritms like Simulated Annealing [10], on the other hand its main drawback consists of "adapting" this strategy to the energy to be minimized. We outline that the contribution of this paper is not a trivial application of $G N C$ to $S F S$ problem. Starting from this kind of energy we have generalized the original formulation of $G N C$, as it will be clearer in the following.

The outline of the paper is as follows. Next section will present some basic concepts about SFS problem useful for understanding the rest of the paper. Section 3 will firstly focus on a general presentation of GNC and then on its application to the energy under study. Some experimental results will be shown and discussed in Section 4.

\section{Shape from Shading}

The aim of Shape from Shading consists of recovering the 3-D shape of an object from 2-D intensity data of an image. The simplest model for image formation is the lambertian one [2/3]. The grey level intensity in correspondence to a given pixel of the image under study can be considered proportional to both the light source $\vec{L}=\left(l_{x}, l_{y}, l_{z}\right)$ and the surface normal $\vec{N}$ intensities. Strictly speaking, the Reflectance Map in the Lambertian model is:

$$
R(p, q)=\max \{0, \rho \vec{N} \cdot \vec{L}\}=\max \left\{0, \rho \frac{l_{z}-p l_{x}-q l_{y}}{\sqrt{1+p^{2}+q^{2}}}\right\}=\max \{0, \rho \cdot \cos (\theta)\}
$$

where $(p, q)=\left(\frac{\partial Z}{\partial x}, \frac{\partial Z}{\partial y}\right)$ is the surface gradient, $\vec{N}=\frac{(-p,-q, 1)}{\sqrt{1+p^{2}+q^{2}}}$ the surface normal, $\rho$ (albedo) is a positive constant including factors such as strength of illumination and surface reflectivity, and $\theta$ is the angle between $\vec{N}$ and $\vec{L}$.

As matter of fact, alternative image formation models have been proposed in literature [11. They try to overcome some intrinsic drawbacks of the lambertian model. Nonetheless, the simplicity of this latter makes it more attractive and effective in many cases.

Hypotheses of uniformity of albedo along with infinite distance between a single light source and the object in the scene are usually made [1].

$S F S$ problem is ill-posed [12] since it consists of recovering the surface quotes $Z(x, y)$ satisfying the image irradiance equation: $E(x, y)=R(p, q)$ with $E(x, y)$ image intensity at position $(x, y)$. Then a smoothness constraint has to be added, yielding the following energy:

$$
\mathcal{E}(p, q, Z)=\underbrace{\sum_{i, j=1}^{N}\left[E_{i, j}-R\left(p_{i, j}, q_{i, j}\right)\right]^{2}}_{\text {Data-Closeness term } \mathcal{E}_{1}}+\underbrace{\lambda \sum_{i, j=1}^{N-1}\left[\Phi\left(\left|\nabla p_{i, j}\right|\right)+\Phi\left(\left|\nabla q_{i, j}\right|\right)\right]}_{\text {Regularization term } \mathcal{E}_{2}}
$$


where $E_{i, j} \quad i, j \in\{1 \ldots N\}$ is the input image, $R\left(p_{i, j}, q_{i, j}\right)$ the lambertian reflectance map, while $\left|\nabla p_{i, j}\right|=\sqrt{\left(p_{i+1, j}-p_{i, j}\right)^{2}+\left(p_{i, j+1}-p_{i, j}\right)^{2}}$ is the gradient of $p$ and similarly for $q$. We selected the well-known function of regularization $\Phi(k)=\frac{k^{2}}{1+\delta k^{2}}$ with $\delta$ positive constant 2310,1314], which performs a selective regularization with preservation of the salient parts of the shape to be recovered [15,16]. $\lambda$ is a positive constant balancing $\mathcal{E}_{1}$ and $\mathcal{E}_{2}$ in (2).

\section{$3 G N C$ for Shape from Shading}

In the previous sections we have introduced $G N C$ as an effective way to minimize discrete non convex energies like the $S F S$ one. Its effectiveness stems from its ability in "avoiding the pitfall of sticking in local minima" (p. 46 of 10]) of a given energy $F$. It is reached by building a finite sequence of energies $F^{(p)}$ with $1 \geq p \geq 0$. The first element $\left(F^{(1)}\right)$ is built as a convex approximation of the original energy and then has a unique (global) minimum. The last one $\left(F^{(0)}\right)$ is the original non convex energy. The intermediate elements of the energies sequence correspond to gradual deconvexed versions of $F^{(0)}$. The main idea of minimization step is very simple. The minimum of $F^{(1)}$ will represent the starting point of minimization of the second element of the sequence and so on. Unfortunately, in spite of a both simple and fast minimization the production of the sequence is often not trivial.

The rest of this section will focus on it. Next subsection will deal with deconvexification of the data-closeness term of (2). It represents a generalization of the classical $G N C$ proposed in 10 . In fact this term has always been convex in all energies minimized so far by this algorithm. This fact will involve some non trivial problems tied to it. On the contrary, the second subsection will focus on the regularization term. Again, also in this case our proposal is more general of that in [10]: it provides a direct solution of the second order energy usually called thin plate.

In order to simplify the computation, in the following it is convenient to consider the functional in (2) in terms of the only variable $z$. Moreover $N \times N$ matrices $z_{i, j}$ will be considered as $N^{2} \times 1$ arrays $z_{k}$, with $k=(i-1) N+j$ to make the formulation tractable.

\subsection{Data-Closeness Term}

The Reflectance Map is:

$$
R_{k}=\frac{l_{z}-l_{x} p_{k}-l_{y} q_{k}}{\sqrt{1+p_{k}^{2}+q_{k}^{2}}}=\frac{l_{z}-l_{x}\left(z_{k+N}-z_{k}\right)-l_{y}\left(z_{k+1}-z_{k}\right)}{\sqrt{1+\left(z_{k+N}-z_{k}\right)^{2}+\left(z_{k+1}-z_{k}\right)^{2}}}
$$

where $p_{k}=\left(\frac{\partial z}{\partial x}\right)_{k}=\left(z_{k+N}-z_{k}\right) \quad$ and $\quad q_{k}=\left(\frac{\partial z}{\partial y}\right)_{k}=\left(z_{k+1}-z_{k}\right)$.

In order to study the convexity of the first term of (2) we have to analyze when its hessian

$$
\frac{\partial^{2} \mathcal{E}_{1}}{\partial z_{i} \partial z_{j}}=2 \cdot \sum_{k} \frac{\partial R_{k}}{\partial z_{i}} \frac{\partial R_{k}}{\partial z_{j}}-\left(E_{k}-R_{k}\right) \frac{\partial^{2} R_{k}}{\partial z_{i} \partial z_{j}} .
$$


is positive definite. We introduce a positive constant $\gamma \in[0,1]$ to build a sequence of reflectance maps whose first term is convex:

$$
\tilde{R}_{k}=\frac{l_{z}-l_{x}\left[\gamma z_{k+N}-z_{k}\right]-l_{y}\left[\gamma z_{k+1}-z_{k}\right]}{\sqrt{1+\gamma\left[\left(z_{k+N}-z_{k}\right)^{2}+\left(z_{k+1}-z_{k}\right)^{2}\right]}} .
$$

In fact, for $\gamma=0$ the function (4) becomes:

$$
\tilde{R}_{k}=l_{z}+z_{k}\left(l_{x}+l_{y}\right)
$$

and then

$$
\frac{\partial^{2} \mathcal{E}_{1}}{\partial z_{i} \partial z_{j}}=2\left(l_{x}+l_{y}\right)^{2} \geq 0 .
$$

On the contrary, for $\gamma \rightarrow 1$, again, we obtain the original lambertian map.

Since for $\gamma \neq 0 \frac{\partial R_{k}}{\partial z_{i}} \neq 0$ only for $k \in\{i, i-1, i-N\}$, it follows:

$$
\begin{aligned}
& \frac{\partial}{\partial z_{i}} \sum_{k}\left(E_{k}-R_{k}\right)^{2}= \\
& -2 \cdot\left[\left(E_{i}-R_{i}\right) \frac{\partial R_{i}}{\partial z_{i}}+\left(E_{i-1}-R_{i-1}\right) \frac{\partial R_{i-1}}{\partial z_{i}}+\left(E_{i-N}-R_{i-N}\right) \frac{\partial R_{i-N}}{\partial z_{i}}\right],
\end{aligned}
$$

and then it is trivial to show that if we derive with respect to variable $z_{j}$ we have:

$$
\frac{\partial^{2}}{\partial z_{i} \partial z_{j}} \sum_{k}\left(E_{k}-R_{k}\right)^{2} \neq 0
$$

only for the indices $j \in\{i, i-1, i+1, i-N, i+N, i-(N-1), i+(N-1)\}$.

\subsection{Regularizing Term}

In this section we will achieve a convex approximation of the regularization term:

$$
\mathcal{E}_{2}=\lambda \sum_{i, j}(\Phi(|\nabla p|)+\Phi(|\nabla q|))
$$

where

$$
|\nabla p|=\sqrt{p_{x}^{2}+p_{y}^{2}}=\sqrt{z_{x x}^{2}+z_{x y}^{2}} \quad, \quad|\nabla q|=\sqrt{q_{x}^{2}+q_{y}^{2}}=\sqrt{z_{x y}^{2}+z_{y y}^{2}} .
$$

and

$$
\begin{aligned}
& z_{x x}(k)=z_{k+N}-2 z_{k}+z_{k-N} \\
& z_{x y}(k)=z_{k+N+1}-z_{k+N}-z_{k+1}+z_{k} \\
& z_{y y}(k)=z_{k+1}-2 z_{k}+z_{k-1} .
\end{aligned}
$$


Considering the circulant matrices $G_{k, l}^{1} G_{k, l}^{2} G_{k, l}^{3}$ generated by

$$
\begin{aligned}
& V^{1}=(\ldots 0,0, \underbrace{1}_{k-N}, 0, \ldots, 0, \underbrace{-2}_{k}, 0, \ldots, 0, \underbrace{1}_{k+N}, 0,0 \ldots) \\
& V^{2}=(\ldots 0,0, \underbrace{1}_{k}, \underbrace{-1}_{k+1}, 0, \ldots, 0, \underbrace{-1}_{k+N}, \underbrace{1}_{k+N+1}, 0,0 \ldots) \\
& V^{3}=(\ldots 0,0,0,0, \underbrace{1}_{k-1}, \underbrace{-2}_{k}, \underbrace{1}_{k+1}, 0,0,0,0 \ldots),
\end{aligned}
$$

the $k-t h$ component of discretized derivative operators can be written as:

$$
z_{x x}(k)=\sum_{l=1}^{N^{2}} G_{k, l}^{1} \cdot z_{l} ; \quad z_{x y}(k)=\sum_{l=1}^{N^{2}} G_{k, l}^{2} \cdot z_{l} ; \quad z_{y y}(k)=\sum_{l=1}^{N^{2}} G_{k, l}^{3} \cdot z_{l} .
$$

Then:

$$
\begin{aligned}
\mathcal{E}_{2}=\frac{\lambda}{\delta^{2}} \sum_{k}\left\{\Psi\left(\delta \sqrt{\left(\sum_{l=1}^{N^{2}} G_{k, l}^{1} z_{l}\right)^{2}+\left(\sum_{l=1}^{N^{2}} G_{k, l}^{2} z_{l}\right)^{2}}\right)\right\}+ \\
+\frac{\lambda}{\delta^{2}} \sum_{k}\left\{\Psi\left(\delta \sqrt{\left(\sum_{l=1}^{N^{2}} G_{k, l}^{2} z_{l}\right)^{2}+\left(\sum_{l=1}^{N^{2}} G_{k, l}^{3} z_{l}\right)^{2}}\right)\right\}
\end{aligned}
$$

where

$$
\Phi(u)=\frac{1}{\delta^{2}} \Psi(\delta u)
$$

Defining the variables:

$$
\begin{aligned}
t_{k} & =\sqrt{\left(\sum_{l=1}^{N^{2}} G_{k, l}^{1} z_{l}\right)^{2}+\left(\sum_{l=1}^{N^{2}} G_{k, l}^{2} z_{l}\right)^{2}} \\
w_{k} & =\sqrt{\left(\sum_{l=1}^{N^{2}} G_{k, l}^{2} z_{l}\right)^{2}+\left(\sum_{l=1}^{N^{2}} G_{k, l}^{3} z_{l}\right)^{2}}
\end{aligned}
$$

(6) can be written as:

$$
\mathcal{E}_{2}(z)=\frac{\lambda}{\delta^{2}} \sum_{k}\left\{\Psi\left(\delta t_{k}\right)+\Psi\left(\delta w_{k}\right)\right\} .
$$

Its convexity derives from the sign of its Hessian $\frac{\partial \mathcal{E}_{2}}{\partial z_{i} \partial z_{j}}>0$.

As regards the first part $\Psi\left(\delta t_{k}\right)$ of the regularization term we have:

$$
\begin{aligned}
& \quad \frac{\partial}{\partial z_{i} \partial z_{j}}\left(\sum_{k} \Psi\left(\delta t_{k}\right)\right)=\sum_{k} \delta^{2} \Psi^{\prime \prime} \cdot \frac{\partial t_{k}}{\partial z_{i}} \cdot \frac{\partial t_{k}}{\partial z_{j}}+\sum_{k} \delta \Psi^{\prime} \cdot \frac{\partial^{2} t_{k}}{\partial z_{i} \partial z_{j}} . \\
& \text { With } A_{k, i}=\left[\left(\sum_{l} G_{k, l}^{1} z_{l}\right) G_{k, i}^{1}+\left(\sum_{l} G_{k, l}^{2} z_{l}\right) G_{k, i}^{2}\right] \text { we have: } \\
& \quad \frac{\partial}{\partial z_{i} \partial z_{j}}\left(\sum_{k} \Psi\left(\delta t_{k}\right)\right)=\sum_{k}\left(A_{k, i} A_{k, j}\right)\left[\delta^{2} \frac{1}{t_{k}^{2}} \Psi^{\prime \prime}\left(\delta t_{k}\right)-\delta \frac{1}{t_{k}^{3}} \Psi^{\prime}\left(\delta t_{k}\right)\right] \\
& \quad+\sum_{k} \delta \frac{1}{t_{k}} \Psi^{\prime}\left(\delta t_{k}\right)\left[G_{k, i}^{1} G_{k, j}^{1}+G_{k, i}^{2} G_{k, j}^{2}\right] .
\end{aligned}
$$


Then

$$
\left[\delta^{2} \frac{1}{t_{k}^{2}} \Psi^{\prime \prime}\left(\delta t_{k}\right)-\delta \frac{1}{t_{k}^{3}} \Psi^{\prime}\left(\delta t_{k}\right)\right]_{\left(\delta t_{k}=u\right)}=\delta^{4}\left[\frac{\Psi^{\prime \prime}(u)}{u^{2}}-\frac{\Psi^{\prime}(u)}{u^{3}}\right]=\delta^{4} \frac{-8}{\left(1+u^{2}\right)^{2}}
$$

bounded as follows $-8 \delta^{4} \leq \frac{-8 \delta^{4}}{\left(1+u^{2}\right)^{2}} \leq 0$ and

$$
\left[\delta \frac{\Psi^{\prime}\left(\delta t_{k}\right)}{t_{k}}\right]_{\left(\delta t_{k}=u\right)}=\delta^{2} \frac{\Psi^{\prime}(u)}{u}=\delta^{2} \frac{2}{\left(1+u^{2}\right)^{2}}
$$

with $0 \leq \frac{2 \delta^{2}}{\left(1+u^{2}\right)^{2}} \leq 2 \delta^{2}$.

Functions (7), (8) can be lower bounded by their minima:

$$
\frac{\partial}{\partial z_{i} \partial z_{j}}\left(\sum_{k} \Psi\left(\delta t_{k}\right)\right) \geq-8 \delta^{4} \sum_{k}\left(A_{k, i} A_{k, j}\right) .
$$

In order to consider the worst case, we analize the maximum of $\sum_{k}\left(A_{k, i} A_{k, j}\right)$. Exploiting eigenvalues for $G^{1}$ e $G^{2}$, we have:

$$
G^{1} z=\gamma^{1} z \Longrightarrow \sum_{l} G_{k, l}^{1} z_{l}=\left(G^{1} z\right)_{k}=\gamma^{1} z_{k} \leq \gamma_{\max }^{1} z_{k}
$$

and similarly for $G^{2}$.

In [17] we proved

$$
\sum_{k} A_{k, i} A_{k, j} \leq C_{z}^{2}\left(\left|\gamma_{\max }^{1}\right|\left|\tilde{\gamma}_{\max }^{1}\right|+\left|\gamma_{\max }^{2} \| \tilde{\gamma}_{\max }^{2}\right|\right)^{2}
$$

with $\gamma_{\max }^{1} \gamma_{\max }^{2}$ and $\tilde{\gamma}_{\max }^{1} \tilde{\gamma}_{\max }^{2}$ respectively the greatest eigenvalues for $G^{1}, G^{2}$ and $\tilde{G}_{i, j}^{1}=\left|G_{i, j}^{1}\right|, \tilde{G}_{i, j}^{2}=\left|G_{i, j}^{2}\right|$ and supposing $z_{k}$ bounded: $z_{k} \leq C_{z}$.

Hence

$$
\frac{\partial}{\partial z_{i} \partial z_{j}}\left(\sum_{k} \Psi\left(\delta t_{k}\right)\right) \geq-8 C_{z}^{2} \delta^{4}\left[\left(\left|\gamma_{\max }^{1} \| \tilde{\gamma}_{\max }^{1}\right|\right)+\left(\left|\gamma_{\max }^{2} \| \tilde{\gamma}_{\max }^{2}\right|\right)\right]^{2}
$$

Similar considerations can be made for the other term containing $\Psi\left(\delta w_{k}\right)$. Then we obtain:

$$
\frac{\partial^{2} \mathcal{E}_{2}}{\partial z_{i} \partial z_{j}} \geq-8 C_{z}^{2} \lambda \delta^{2} \Gamma_{\max }
$$

with

$$
\Gamma_{\max }=\left(\left|\gamma_{\max }^{1}\right|\left|\tilde{\gamma}_{\max }^{1}\right|+\left|\gamma_{\max }^{2}\right|\left|\tilde{\gamma}_{\max }^{2}\right|\right)^{2}+\left(\left|\gamma_{\max }^{2}\right|\left|\tilde{\gamma}_{\max }^{2}\right|+\left|\gamma_{\max }^{3}\right|\left|\tilde{\gamma}_{\max }^{3}\right|\right)^{2}
$$

Eigenvalues have been computed as in Appendix D of [10 with suitable changes. The obtained constraints are general and can be applied to all energies having the first term convex and the same regularization function. 


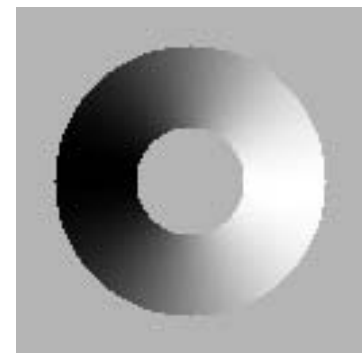

a)

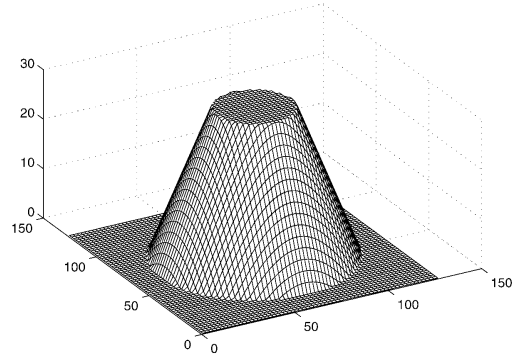

b)

Fig. 1. The $128 \times 128$ original discrete shape: a)gray-scale image, b) surface.

\subsection{Implementation}

From the data-closeness term of the energy $\mathcal{E}$ we have:

$$
\frac{\partial \mathcal{E}_{1}}{\partial z_{i} \partial z_{j}}=2\left(l_{x}+l_{y}\right)^{2}
$$

The Hessian matrix is positive definite if the following condition is satisfied:

$$
\Gamma_{\max } \leq \frac{\left(l_{x}+l_{y}\right)^{2}}{4 C_{z}^{2} \lambda \delta^{2}}
$$

Then under the above constraint, the functional (21) is convex.

Now we set

$$
\delta_{p}^{2}=\frac{\left(l_{x}+l_{y}\right)^{2}}{4 p C_{z}^{2} \lambda \Gamma_{\max }} \quad \text { and } \quad \gamma_{p}=\frac{1-p}{1-\bar{p}}
$$

with $1 \geq p \geq \bar{p}$, where $\bar{p}=\frac{\left(l_{x}+l_{y}\right)^{2}}{4 C_{z}^{2} \lambda \delta^{2} \Gamma_{\max }}$.

Let us denote $\mathcal{E}^{(p)}$ the energy in (2) corresponding to the values $\delta_{p}$ and $\gamma_{p}$. The sequence of energies is such that $\mathcal{E}^{(1)}$ is convex and $\mathcal{E}^{(p)}$ changes towards $\mathcal{E}$ as $\mathrm{p}$ approaches $\bar{p}$. We first minimize the energy $\mathcal{E}^{(1)}$ and then the parameter $p$ is gradually lowered in the interval $1 \searrow \bar{p}$.

\section{Experimental Results and Discussion}

We have applied our model on many shapes. In this paper we will show the results on the shape depicted in Fig. 1 since simple but with evident discontinuities. Fig.2 shows the surface recovered by our model in just 350 seconds on a Risk work station Octane/SI R10000 175MHz/1Mb cache, considering a multigrid speed up in the convex step. It can be noticed that our model is able to catch discontinuities usually difficult to reach by SFS minimization models. In other words our minimization model strongly decreases the energy achieving good results. To better understand this point, we compared our performances 

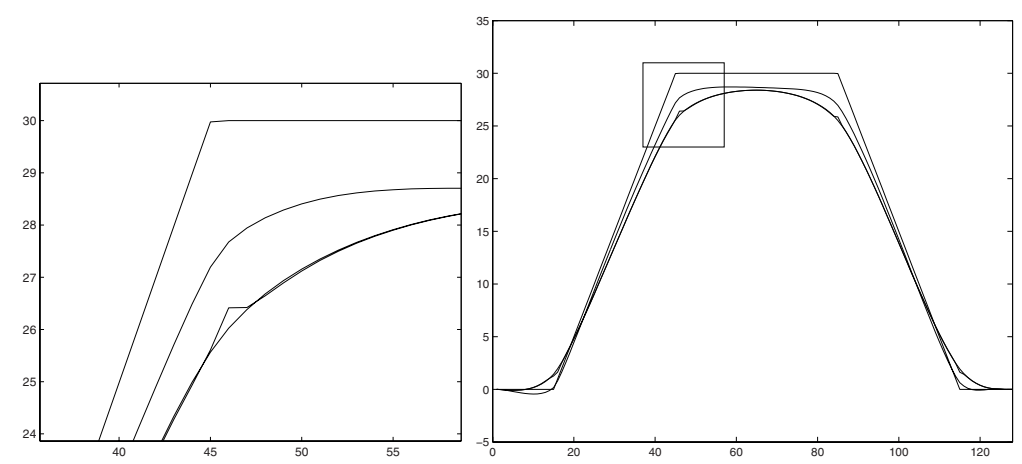

Fig. 2. Row n. 64 of: original surface (top most), convex recovery (bottom most), GNC (close to the convex one but with discontinuities), Gauss-Seidel (intermediate solution). A zoom around a discontinuity point is also evidenced.

with the ones achieved by another well-known energy minimization: Gauss-Seidel algoritm on Euler equations relative to $p, q, Z$. In order to make comparable the performances of both models, we start from the same convex surface obtained via the convex energy relative to (2), i.e. with $\Phi=t^{2}$. This choice accounts for the same speed up in both minimizations in the respective convex steps via a multigrid model [18]. Looking again at Fig. 2 we can understand the difference between the behavior of the two minimizations. Our solution remains close to the convex surface outperforming the discontinuity regions. The other one tries to reach the original surface but it loses the discontinuity information in opposition to non convex models aim.

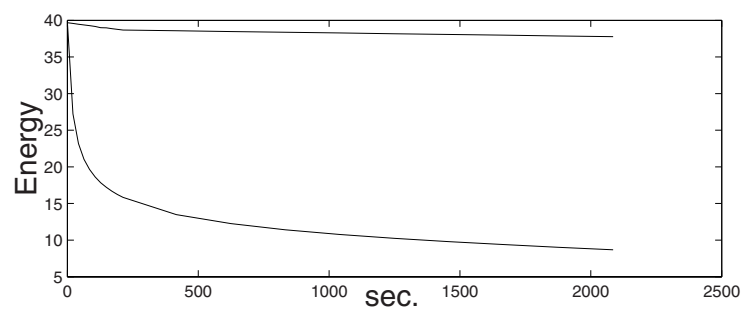

Fig. 3. Energy decay behavior during minimization performed by Gauss-Seidel (top) and GNC (bottom).

Fig. 3 emphasizes this fact showing the energy decay versus time of both models. Although its closeness to the convex solution, our model makes a drastic reduction of the global energy that entails a better recovering of the discontinuity points. On the contrary, the other minimization shows a compensation between a better surface (closer to the original one) and the lost of discontinuities. Moreover, our model achieves a good result in a few seconds against many minutes of the other. Finally, in order to show the potentialities of our approach, in Fig. 4 

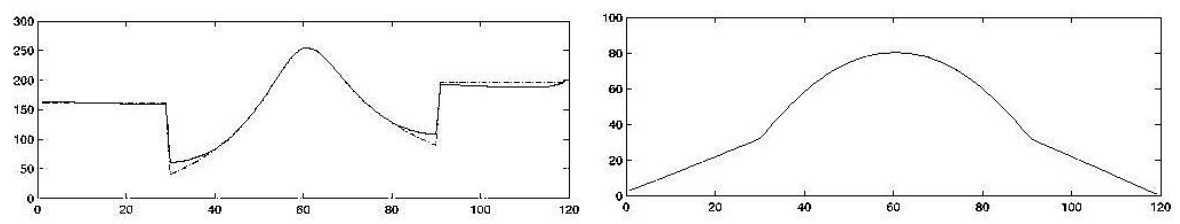

Fig. 4. An example of $G N C$ performed on a 1-D case: on the left original (dashed) and recovered (solid) reflectance map, on the right original and recovered surfaces (nearly coincident).

we have shown the result obtained in 180 seconds on a 1-D version of the energy in (2) where the original surface is now a cone overlapped on a spherical cap. It turns out that paying a higher computational time, our approach can achieve results close to absolute minimum. In order to maintain the time moderate a further speed up on the non convex steps should be achieved. This is presently an open problem and will be the topic of our future research.

\section{Acknowledgements}

Authors would like to thank dr. Riccardo March for his helpful suggestions to develop this work.

\section{References}

1. Pentland, A.: Shape information from shading:a theory about human perception. In: Proceedings of Int'l Conference on Computer Vision. (1988) 404-413

2. Zhang, R., Tsai, P., Cryer, J., Shah, M.: Shape from shading: a survey. IEEE Transactions on Pattern Analysis and Machine Intelligence 21 (1999) 690-705

3. Ikeuchi, K., Horn, B.: Numerical shape from shading and occluding boundaries. Artificial Intelligence 17 (1981) 141-184

4. Zeng, Q., Chellappa, R.: Estimation of illuminant direction, albedo, and shape from shading. IEEE Transactions on Pattern Analysis and Machine Intelligence 13 (1991) 680-702

5. Bruckstein, A.M.: On shape from shading. Computer vision, Graphics, and Image Processing 44 (1988) 139-154

6. Rouy, E., Tourin, A.: A viscosity solution approach to shape from shading. SIAM Journal of Numerical Analysis 29 (1992) 867-884

7. Kimmel, R., Sethian, J.: Optimal algorithm for shape from shading and path planning. Journal of Mathematical Imaging and Vision 14 (2001) 237-244

8. Pentland, A.: Local shading analysis. IEEE Transactions on Pattern Analysis and Machine Intelligence 6 (1984) 170-187

9. Tsai, P., Shah, M.: Shape from shading using linear approximation. Image and Vision Computing 12 (1993) 487-498

10. Blake, A., Zisserman, A.: Visual Reconstruction. MIT Press, Cambridge MA (1987) 
11. Stewart, A., Langer, M.: Towards accurate recovery of shape from shading under diffuse lighting. IEEE Transactions on Pattern Analysis and Machine Intelligence 19 (1997) 1020-1025

12. Hadamard, J.: Lectures on the Cauchy Problem in Linear Partial Differential Equations. Yale University Press, (1923)

13. Mumford, D., Shah, J.: Optimal approximation by piecewise smooth functions and associated variational problems. Communications on Pure and Applied Mathematics 42 (1989) 577-685

14. March, R.: Visual reconstruction with discontinuities using variational methods. Image and Vision Computing 10 (1992) 30-38

15. Chipot, M., March, R., Vitulano, D.: Numerical analysis of oscillations in a non convex problem related to the image selective smoothing. Journal of Computational and Applied Mathematics 136 (2001) 123-133

16. Tebould, S., Aubert, L.B.F.G., Barlaud, M.: Variational approach for edge preserving regularization using coupled pde's. IEEE Transactions on Image Processing 7 (1998) 387-397

17. Gelli, D., Vitulano, D.: Gnc for thin plate. Technical Report Q33-002, IAC-C.N.R. (2002)

18. Terzopoulos, D.: Image analysis using multigrid relaxation methods. IEEE Trans. PAMI 8 (1986) 129-139 